\title{
Repeatable antibiotic prescriptions: an assessment of patient
}

\section{attitudes, knowledge and advice from health professionals}

\author{
Angus Thompson ${ }^{1}$, Shannan Copping 2 , Andrew Stafford ${ }^{3}$ and Gregory Peterson ${ }^{1}$
}

1. School of Pharmacy, University of Tasmania, Hobart, Tasmania; 2. Kings Meadows Pharmacy, Launceston,

Tasmania; 3. School of Pharmacy, Curtin University, Perth, Western Australia

\section{RESEARCH}

Please cite this paper as: Thompson A, Copping S, Stafford A and Peterson G. Repeatable antibiotic prescriptions: an assessment of patient attitudes, knowledge and advice from health professionals. AMJ 2014, 7, 1, 1-5. http://doi.org/10.21767/AMJ.2014.1871.

\section{Corresponding Author:}

Angus Thompson

Locked Bag 26, University of Tasmania, Hobart

Tasmania 7001.

Email: Angus.thompson@utas.edu.au

\section{Abstract}

\section{Background}

Previous Australian research has identified that general practice software systems appear to be associated with an increase in repeatable antibiotic prescriptions. Such prescriptions potentially facilitate the use of antibiotics without medical consultation and may be inconsistent with attempts to promote prudent use of antimicrobials.

\section{Aims}

We sought to assess knowledge and attitudes to antibiotics amongst patients presenting with a repeatable prescription; and the provision of supporting advice from healthcare professionals regarding use of these repeats.

\section{Method}

Six community pharmacies across Tasmania invited patients presenting with a repeatable antibiotic prescription to participate in the study. Participants were asked to complete a questionnaire and return this to the research team in a pre-paid envelope.

\section{Results}

Fifty-seven of 244 (23 per cent) surveys were returned. Regarding provision of advice on use of the repeat, 14 (25 per cent) of respondents stated that they were given no advice by the prescriber and 19 (30 per cent) no advice from the pharmacist. Five ( 9 per cent) were given no advice from either prescriber or pharmacist. One-third of respondents indicated that they would keep the repeat for future use and around three-quarters perceived no major safety concerns with antibiotics.

\section{Conclusion}

Further research is needed, however, this small study suggests that provision of information to patients regarding appropriate use of repeatable antibiotic prescriptions is suboptimal. This coupled with existing patient knowledge and attitudes may contribute to inappropriate use of antibiotics.

\section{Key Words}

Antibiotic, prescription, repeat, patient, knowledge, attitude

\section{What this study adds:}

\section{What is known about this subject?}

Australian general practice prescriptions for antibiotics commonly include a repeat and the use of electronic prescribing software may contribute to this practice.

\section{What new information is offered in this study?}

Provision of information to patients regarding appropriate use of repeatable antibiotic prescriptions is sub-optimal and this coupled with existing patient knowledge and attitudes may contribute to inappropriate use of antibiotics.

3. What are the implications for research, policy or practice?

Repeatable antibiotic prescriptions should only be issued when they are clinically indicated and supported by clear advice on their appropriate use.

\section{Background}

Inappropriate and indiscriminate use of antimicrobials provides favourable conditions for resistant microorganisms to emerge and spread. ${ }^{1}$ Tackling antimicrobial resistance is 
increasingly recognised as a priority area for healthcare systems globally, as our ability to effectively treat infection and achieve health gains through medical advances such as chemotherapy, transplantation, and complex surgery is threatened. ${ }^{1}$

Previous research has identified that Australian general practice prescribing software systems often automatically default to issuing antibiotic prescriptions with the maximum number of repeats allowed by the Pharmaceutical Benefits Scheme and that this is associated with increased prescribing of repeats. ${ }^{2,3}$ In Australia, as with many other developed countries, the regulatory system restricts access to antibiotics through prescription-only status. However, antibiotic prescriptions with repeats have the potential to undermine this. There are several reasons why repeatable antibiotic prescriptions may be of concern. Firstly, they provide patients with an opportunity to extend their own initial course, which may be clinically inappropriate. Secondly, patients have a prescription that they can retain for use within the next 12 months for any suspected infection they experience. Furthermore, the repeat prescription could be used for a third party, for example, a family member with similar symptoms; this carries additional risks relating to potential adverse drug events and drug interactions. In all cases, issuing antibiotic prescriptions with repeats effectively sanctions use of antibiotics without the requirement for medical consultation.

The risk that these scenarios will translate into inappropriate use of antibiotics may be dependent on patients having a poor understanding, knowledge, and attitude towards the use of antibiotics and repeat prescriptions for antibiotics in particular. However, there is currently little in the published literature on this subject. This pilot study aimed to address these gaps in our understanding. The objective was to determine the frequency with which patients received advice from a doctor or pharmacist regarding the appropriate use antibiotic repeats and to assess patients' existing levels of knowledge and attitudes to antibiotics and their repeat prescriptions.

\section{Method}

Eleven Tasmanian community pharmacies were invited to take part in the study and of these, six agreed to participate. Survey packages containing an information sheet for patients, a consent form, knowledge and attitudes questionnaire, a form to enter a draw for a shopping voucher (offered to incentivise response), and a pre-paid envelope for return of the questionnaire, were distributed to the six pharmacies.
Participating pharmacies invited patients presenting an original prescription for an oral antibiotic with a repeat to take part in the study. Patients prescribed a long-term antibiotic (such as doxycycline for acne) were excluded, as the standard treatment regimen requires the use of repeats. Pharmacists were asked to indicate if the prescription was handwritten or computer-generated and to attach a duplicate dispensing label to the questionnaire form, with all patient and prescriber identifiers removed, in order that details of the prescribed antibiotic could be recorded.

Patients were asked to indicate what information they had been given by their doctor or pharmacist regarding use of the repeat. Specifically, whether they were advised to only use the first pack of antibiotics, to take both packs of antibiotics or what to do with the repeat prescription if it was not needed. Patients were also provided with a free text section in which they could provide details of any other information they received regarding use of the repeat from either their doctor or pharmacist.

The questionnaire asked patients to score their level of agreement with the following statements using a five-point Likert Scale:

- Antibiotics are completely safe.

- Antibiotics have no side effects.

- Antibiotics should also be available without a prescription.

- If I have taken an antibiotic once, I should be able to get it again without a prescription.

- Antibiotics work against viral infections (such as influenza/colds).

- Antibiotics work against bacterial infections.

- Overusing antibiotics reduces how well they work.

- Repeat antibiotic prescriptions should be filled/collected and used immediately after finishing the first course.

- Antibiotic repeats can be used for family/friends who have a similar infection.

- I can keep my repeat antibiotic prescription for next time I am unwell.

In order to analyse participants' responses to the knowledge and attitudes survey, the most appropriate answers were classified as Correct, while the inappropriate responses were classified as Incorrect. These are summarised in Table 1).

The study was approved by the Human Research Ethics Committee (Tasmania) Ref No. H11585 prior to its commencement. 
Table 1: Responses to knowledge $\&$ attitude questionnaire

\begin{tabular}{|c|c|c|c|c|c|}
\hline $\begin{array}{l}\text { Strongly } \\
\text { disagree }\end{array}$ & Disagree & Neutral & Agree & $\begin{array}{c}\text { Strongly } \\
\text { agree }\end{array}$ & Unsure \\
\hline \multicolumn{6}{|c|}{ (1) Antibiotics are completely safe. } \\
\hline $\begin{array}{c}2 \\
(3.5 \%)\end{array}$ & $\begin{array}{c}14 \\
(24.6 \%)\end{array}$ & $\begin{array}{c}13 \\
(22.8 \%)\end{array}$ & $\begin{array}{c}17 \\
(29.8 \%)\end{array}$ & $\begin{array}{c}8 \\
(14.0 \%)\end{array}$ & $\begin{array}{c}3 \\
(5.3 \%)\end{array}$ \\
\hline \multicolumn{6}{|c|}{ (2) Antibiotics have no side effects. } \\
\hline $\begin{array}{c}9 \\
(15.8 \%)\end{array}$ & $\begin{array}{c}35 \\
(61.4 \%)\end{array}$ & $\begin{array}{c}6 \\
(10.5 \%)\end{array}$ & $\begin{array}{c}3 \\
(5.3 \%)\end{array}$ & $\begin{array}{c}1 \\
(1.7 \%)\end{array}$ & $\begin{array}{c}3 \\
(5.3 \%)\end{array}$ \\
\hline \multicolumn{6}{|c|}{ (3) Antibiotics should also be available without a prescription. } \\
\hline $\begin{array}{c}30 \\
(52.6 \%)\end{array}$ & $\begin{array}{c}22 \\
(38.6 \%)\end{array}$ & $\begin{array}{c}2 \\
(3.5 \%)\end{array}$ & $\begin{array}{c}0 \\
(0 \%)\end{array}$ & $\begin{array}{c}3 \\
(5.3 \%)\end{array}$ & $\begin{array}{c}0 \\
(0 \%)\end{array}$ \\
\hline
\end{tabular}

(4) If I have had an antibiotic once, I should be able to get it again without a prescription.

\begin{tabular}{|c|c|c|c|c|c|}
\hline $\mathbf{2 4}$ & $\mathbf{2 4}$ & 3 & 2 & 4 & 0 \\
$\mathbf{( 4 2 . 1 \% )}$ & $\mathbf{( 4 2 . 1 \% )}$ & $(5.3 \%)$ & $(3.5 \%)$ & $(7.0 \%)$ & $(0 \%)$ \\
\hline
\end{tabular}

(5) Antibiotics work against viral infections (such as influenza/cold).

\begin{tabular}{|c|c|c|c|c|c|}
\hline $\begin{array}{c}\mathbf{1 8} \\
\mathbf{( 3 1 . 6 \% )}\end{array}$ & $\begin{array}{c}\mathbf{2 2} \\
\mathbf{( 3 8 . 6 \% )}\end{array}$ & $\begin{array}{c}7 \\
(12.3 \%)\end{array}$ & $\begin{array}{c}5 \\
(8.8 \%)\end{array}$ & $\begin{array}{c}1 \\
(1.7 \%)\end{array}$ & $\begin{array}{c}4 \\
(7.0 \%)\end{array}$ \\
\hline \multicolumn{6}{|c|}{ (6) Antibiotics work against bacterial infections. } \\
\hline 1 & 1 & 3 & $\mathbf{3 3}$ & $\mathbf{1 7}$ & 2 \\
$(1.7 \%)$ & $(1.7 \%)$ & $(5.3 \%)$ & $\mathbf{( 5 7 . 9 \% )}$ & $\mathbf{( 2 9 . 8 \% )}$ & $(3.5 \%)$ \\
\hline
\end{tabular}

(7) Overusing antibiotics reduces how well they work.

\begin{tabular}{|c|c|c|c|c|c|}
\hline 2 & 3 & 2 & $\mathbf{2 7}$ & $\mathbf{1 9}$ & 4 \\
$(3.5 \%)$ & $(5.3 \%)$ & $(3.5 \%)$ & $(\mathbf{4 7 . 4 \% )}$ & $\mathbf{( 3 3 . 3 \% )}$ & $(7.0 \%)$ \\
\hline
\end{tabular}

(8) Repeat antibiotic prescriptions should be filled/collected and used immediately after finishing the first course.

\begin{tabular}{|c|c|c|c|c|c|}
\hline 3 & 11 & 9 & $\mathbf{2 1}$ & $\mathbf{9}$ & 4 \\
$(5.3 \%)$ & $(19.3 \%)$ & $(15.8 \%)$ & $(\mathbf{3 6 . 8 \% )}$ & $\mathbf{( 1 5 . 8 \% )}$ & $(7.0 \%)$ \\
\hline
\end{tabular}

(9) Antibiotic repeats can be used for family/friends who have a similar infection.

\begin{tabular}{|c|c|c|c|c|c|}
\hline 36 & $\mathbf{2 0}$ & 0 & 0 & 0 & 1 \\
$\mathbf{( 6 3 . 2 \% )}$ & $\mathbf{( 3 5 . 1 \% )}$ & $(0 \%)$ & $(0 \%)$ & $(0 \%)$ & $(1.7 \%)$ \\
\hline
\end{tabular}

(10) I can keep my repeat antibiotic prescription at home for next time I am unwell.

\begin{tabular}{|c|c|c|c|c|c|}
\hline $\mathbf{2 1}$ & $\mathbf{1 9}$ & 11 & 4 & 1 & 1 \\
$\mathbf{( 3 6 . 8 \% )}$ & $\mathbf{( 3 3 . 3 \% )}$ & $(19.3 \%)$ & $(7.0 \%)$ & $(1.7 \%)$ & $(1.7 \%)$ \\
\hline
\end{tabular}

Note: Responses classified as correct for the purposes of analysis are highlighted in bold.

\section{Results}

The six pharmacies that agreed to participate represented the North and South of Tasmania, as well as urban and rural locations. Of the 244 survey packages distributed to patients over a 2-month period, 57 responses were returned to the research team, equating to a response rate of 23 per cent.

Eleven different oral antibiotics were prescribed with repeats in this study, with four of these (amoxycillin, amoxycillin/clavulanate, cephalexin, and roxithromycin) accounting for 43 (75 per cent) of the prescriptions. Participants were asked to indicate the type of infection that was being treated and the most common responses were: chest infection/pneumonia 11 (19 per cent), sinus/nose infection 11 (19 per cent), urinary tract infection 9 (16 per cent), and ear infection 6 (10 per cent). Of the 11 repeatable prescriptions for chest infections/pneumonia, 8 were for either amoxycillin/clavulanate or roxithromycin.
Forty-three of the 57 responses were marked to indicate whether prescriptions were computer-generated or handwritten, with 41 (95 per cent) of these being computergenerated.

Table 1 summarises the responses considered to be correct for each statement and the number of correct responses received from study participants for each of these statements.

Regarding provision of advice on the intended use of the repeat prescription, 14 participants ( 25 per cent) stated that they received no information from their doctor, and 17 (30 per cent) were given no information by their pharmacist. Five participants ( 9 per cent) stated that they received no advice from either their doctor or pharmacist.

\section{Discussion}

As one of the highest users of antibacterials in the developed world, it has been suggested that Australia needs a more judicious approach to antibiotic use. ${ }^{4}$ Antibiotic stewardship is becoming increasingly established in the hospital setting, but it has recently been recommended that stewardship activities be extended into the community. ${ }^{4}$ While it is likely that there would be many aspects to community-based stewardship programs, it is logical that attention would be paid to repeatable antibiotic prescriptions.

This small study provides a number of potentially valuable findings relevant to the prescription of antibiotics with repeats. Advice about the use of antibiotic repeats from either a prescriber or pharmacist was provided to the majority of participants. However, 25 per cent and 30 per cent patients recalled that they received no advice from their doctor and pharmacist, respectively, and 9 per cent received no advice from either. Given the large volume of repeatable antibiotic prescriptions issued in Australia each year, it may be implied from the results of this study that many thousands of patients are provided with a repeat yet receive no supporting advice regarding its appropriate use.

The primary concern arising from this is an increased use of antibiotics and the associated growth in resistance. However, there are also implications given that the initial course (typically 3 to 7 days) should be sufficient for the desired response in many common infections in primary care and this is consistent with recommendations in Australian guidelines. ${ }^{5}$ Prolonged courses may increase the risk of patients experiencing adverse effects, or lead to a delay in seeking medical advice for a sub-optimally responding infection, which may have clinically significant consequences. While this study did not aim to assess whether antibiotic therapy was concordant with guidelines, 
it is important to acknowledge that where there is nonconcordance, the use of repeatable prescriptions may be particularly undesirable. Examples identified in our study were the use of repeatable antibiotic prescriptions for amoxycillin/clavulanate or roxithromycin to treat chest infections/pneumonia. Neither of these antibiotics is recommended by the current Australian guidelines for the out-patient treatment of community-acquired pneumonia or acute exacerbation of chronic obstructive pulmonary disease (COPD). ${ }^{5}$

It should be acknowledged that judicious use of repeatable prescriptions might be appropriate for some patients. One example of this would be for patients with COPD, in whom timely commencement of guideline-recommended therapy at the onset of an exacerbation may be desirable as part of a COPD action plan. Situations such as this should, however, be seen as the exception rather than the rule.

Responses to the knowledge and attitudes survey indicated wide variation in the level of knowledge across the different themes. Overall, the responses were generally encouraging, with an appropriate answer being given by the majority of participants to 9 out of 10 questions. The highest level of appropriate response (98 per cent) was to the question regarding retaining repeats and using them for family and friends. This is particularly reassuring given the multiple risks associated with this behaviour. However, the responses in two areas in particular, warrant further comment. In contrast to the overwhelmingly appropriate response to the question about future use for a third party, only 67 per cent of respondents expressed disagreement with the statement regarding retaining repeats for their own use in the future. This means that around one-third of patients with repeats would potentially self-diagnose and initiate antibiotic therapy without medical advice. Problems relating to this could include: recurrent symptoms that may not be related to infection or could be the result of a viral rather than bacterial infection; that the previous course may have led to selective survival of resistant bacteria; and that the patient may use the repeat to treat a different type of infection where the antibiotic would not be expected to be effective.

The lowest "correct" response rate of 25 per cent was to the question regarding antibiotic safety. While this may reflect the generally good tolerability and safety of the majority of antibiotics in clinical use, it is of concern that around three-quarters of respondents did not recognise that antibiotics can be associated with adverse effects, which may include hypersensitivity, nausea, vomiting, diarrhoea, and candidiasis. Furthermore, antibiotics may be associated with potentially serious drug interactions, for example, most antibacterials interact with warfarin; and those macrolides that inhibit CYP3A4 (in particular erythromycin and clarithromycin) are involved in clinically significant interactions with a wide range of commonly prescribed drugs, increasing the risk of adverse effects and toxicity. An additional consideration regarding repeatable antibiotic prescriptions is that if advice is provided by a pharmacist, it may be inconsistent with the intentions of the prescriber and lead to confusion for patients.

Organisations such as NPS MedicineWise have already implemented initiatives to improve public knowledge regarding the value of antibiotics and how these can be preserved. However, the findings of this study may help inform the future direction and development of such initiatives.

Respondents were drawn from a range of areas across Tasmania and as such the results may be generalisable to the wider Australian population; however, there are limitations to this study. These include the small sample size and low response rate. Failure to report advice being provided may reflect patients simply not remembering this at the time of completing the questionnaire, rather than actual lack of advice provision. It would have also been useful to survey doctors to determine whether the prescribing of antibiotics with a repeat was intentional or the result of failure to manually override a default setting in their clinical software.

\section{Conclusion}

It appears that the potential adverse consequences of issuing repeat prescriptions for antibiotics may be compounded by gaps in the provision of advice to patients by prescribers and pharmacists. Patient knowledge and attitudes regarding many aspects of antibiotics were generally appropriate amongst the study population. Nonetheless, inappropriate perceptions regarding the safety of antibiotics and the significant proportion of patients who would self-treat with repeats in the future are factors that make the widespread practice of issuing repeat antibiotic prescriptions undesirable. Future initiatives to educate patients regarding appropriate use of antibiotics, should address these particular areas.

This study adds to our understanding of patient attitudes and knowledge regarding repeatable antibiotic prescriptions. However, further research may help determine the way in which repeatable antibiotic prescriptions are actually used, for example, to extend initial courses or treat separate episodes of suspected infection at a later date.

In the meantime, prescribers should ensure that a repeat prescription is only authorised for an antibiotic when it is 
clinically indicated, adjusting default settings in prescribing software would be one way to encourage this. Finally, prescribers and pharmacists should ensure that patients receive clear, consistent and appropriate information about how to use any repeat that has been issued.

\section{References}

1. World Health Organisation. Combat antimicrobial resistance: fact sheet 2011 [Internet]. Available from: http://www.who.int/world-health-day/2011/en/index.html.

2. Newby DA, Fryer JL, Henry DA. Effect of computerised prescribing on use of antibiotics. Med J Aust. 2003 Mar 3;178(5):210-3.

3. Newby DA, Robertson J. Computerised prescribing: assessing the impact on prescription repeats and on generic substitution of some commonly used antibiotics. Med J Aust. 2010 Feb 15;192(4):192-5.

4. McKenzie D, Rawlins M, Del Mar C. Antimicrobial stewardship: what's it all about? Australian Prescriber 2013; 36: 116-20.

5. Therapeutic Guidelines - Antibiotic (version 14). Therapeutic Guidelines, Melbourne; June 2010

\section{ACKNOWLEDGEMENTS}

The research team acknowledge the assistance of participating community pharmacists and patients, without whose cooperation this research would not have been possible.

\section{PEER REVIEW}

Not commissioned. Externally peer reviewed.

\section{CONFLICTS OF INTEREST}

The authors declare that they have no competing interests.

\section{FUNDING}

No external funding received; this research was funded wholly from the budget allocated to the BPharm (Hons) program at the School of Pharmacy, University of Tasmania.

\section{ETHICS COMMITTEE APPROVAL}

Approved by the Human Research Ethics Committee (Tasmania) Ref No. H11585 prior to its commencement. 